\title{
Misinterpretation of glioblastoma as ADEM: potentially harmful consequences of over-diagnosis of COVID-19 vaccine-associated adverse events
}

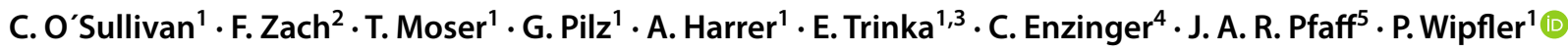

Received: 1 July 2021 / Revised: 7 July 2021 / Accepted: 8 July 2021 / Published online: 24 July 2021

(c) Springer-Verlag GmbH Germany, part of Springer Nature 2021

Dear Sirs,

As of June 15th 2021, the COVID-19 pandemic, caused by the novel coronavirus severe acute respiratory syndrome coronavirus 2 (SARS-CoV-2), has resulted in more than 177 million confirmed cases worldwide and more than 3.8 million deaths [1]. Although respiratory disease represents the most common and important presentation, reports of neurological features have been increasingly described [2].

Among the reported neurological manifestations are cases of acute disseminated encephalomyelitis (ADEM), a rare immune-mediated demyelinating disease [3-5]. As ADEM is associated with vaccinations as well as with viral infection, particular vigilance was paid to the recognition of the diverse clinical manifestations of ADEM following introduction of the initial messenger RNA (mRNA) vaccines.

Early recognition of the neurological sequelae of newly introduced vaccinations remains of paramount importance. Nevertheless, we must also remain on alert that the current level of resources concentrated on COVID-19-related symptoms does not divert a disproportionate amount of

P. Wipfler

p.wipfler@salk.at

1 Department of Neurology, Christian Doppler University Hospital, Paracelsus Medical University and Center for Cognitive Neuroscience, Ignaz-Harrer-Straße 79, 5020 Salzburg, Austria

2 Department of Neurology, Kardinal Schwarzenberg Hospital, Schwarzach, Austria

3 Institute for Neuroscience, Christian Doppler University Hospital, Paracelsus Medical University and Center for Cognitive Neuroscience Salzburg, Salzburg, Austria

4 Department of Neurology, Medical University of Graz, Graz, Austria

5 Department of Neuroradiology, Christian Doppler University Hospital, Paracelsus Medical University, Salzburg, Austria focus from more frequent neurological conditions. We here thus present an ADEM mimic occurring subsequent to the administration of the COVID mRNA vaccination.

A previously healthy 31-year-old female first noted a slight weakness of her right leg about 7 days after receiving the first dose of a COVID-19 mRNA vaccine (Comirnaty ${ }^{\circledR}$ BioNTech Manufacturing GmbH, Germany). She initially reported slight drowsiness and headache without fever following vaccination, which resolved within $24 \mathrm{~h}$.

Following the administration of the second intramuscular dose of the vaccination, 21 days after the first, the preexisting weakness of the right leg rapidly worsened and was accompanied by severe headache and night chills. Neurological examination on day 28 showed a mild central paresis of the right leg and numbness of the plantar surface of the foot. Babinski sign was negative bilaterally. Initial brain magnetic resonance imaging (MRI) revealed three T2 hyperintense lesions with a diameter of approximately around $2.5 \mathrm{~cm}$ in the left frontoparietal periventricular white matter. The lesions were also hyperintense on diffusion-weighted imaging with ADC restriction, and the most dorsal lesion demonstrated gadolinium ring enhancement (Fig. 1). MRI of the spine yielded no evidence of demyelinating lesions.

Cerebrospinal fluid (CSF) diagnostics revealed a normal cell count, no blood/CSF barrier disruption and no evidence of intrathecal IgG synthesis. Oligoclonal bands, CSF and serum antibodies including Aquaporin-4, myelin oligodendrocyte glycoprotein (MOG), and the autoimmune encephalitis profile were negative. MOG-IgG is associated with several atypical demyelinating syndromes, more frequently in children than in adults [6]. However, antibody negativity does not exclude the diagnosis of ADEM. AntiSARS-CoV-2 antibodies were excessively high indicating a pronounced immune response after vaccination.

Following the exclusion of alternate differential diagnoses, we initially considered ADEM as the most likely 


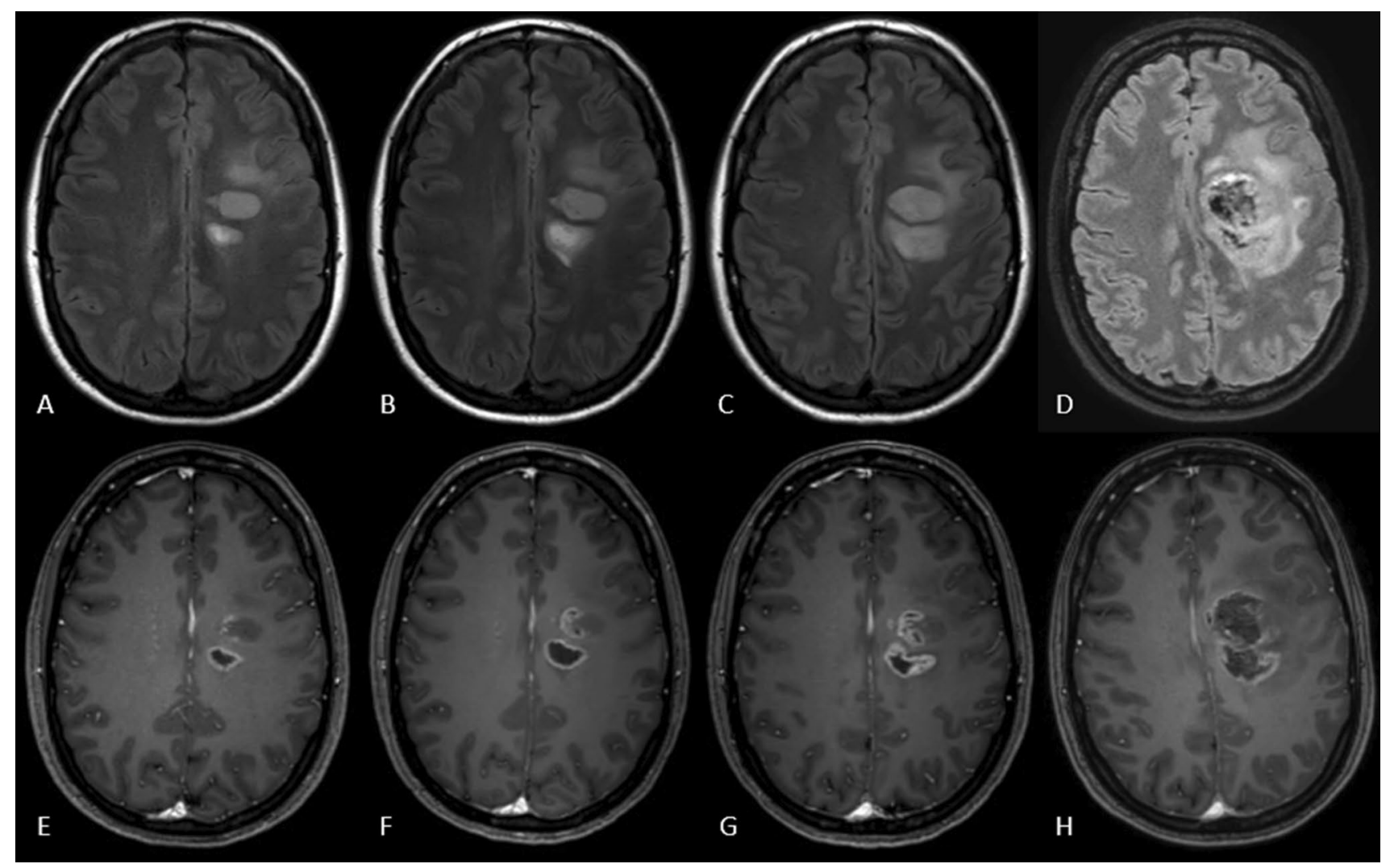

Fig. 1 Axial FLAIR (A-D) and T1-weighted Gd-enhanced MR images (E-F) acquired 40 days (A and E), 49 day (B and $\mathbf{F}$ ), 72 days (C and $\mathbf{G}$ ) and 93 days (D and $\mathbf{H}$ ) following the date of administration of the first dose of a COVID-19 mRNA vaccine. Note the change from multifocal ovoid to large, flocculent FLAIR hyperintensities

diagnosis, based on MRI findings, the clinical presentation and the history of recent vaccination.

The patient received high-dose intravenous methylprednisolone (IVMP) $(1000 \mathrm{mg} / \mathrm{d})$ for 5 days. We observed a full recovery of muscle strength after this first course.

However, the patient again presented at our emergency department with a progression of neurological symptoms (pronounced dysarthria and right upper limb weakness) 3 weeks after discharge. Appearance of new lesions at MRI suggested the diagnosis of multiphasic ADEM (Fig. 1). Following plasmapheresis, no symptomatic improvement was noted, which prompted a reevaluation of the suspected diagnosis. The follow-up MRI (day 72, Fig. 1C, G) showed an increase in the intensity and breadth of the contrast enhancement, i.e., from a ring enhancement to an irregular rind. Simultaneously new microbleeds occurred suggesting necrosis compared with the MRI performed on day 49 (Fig. 1B, F). The patient was referred to neurosurgery for biopsy of the frontoparietal lesion. Histological findings were in keeping with a glioblastoma multiforme. with peripheral punctate and ring enhancement ( $\mathbf{A}$ and $\mathbf{E})$ mimicking an acute disseminated encephalomyelitis (ADEM) to a heterogeneous, hyperintense mass with adjacent vasogenic edema and an irregular rind of enhancement surrounding a central necrosis (D and $\mathbf{H})$ characteristic of a glioblastoma multiforme (GBM)

We believe that this case presents a note of caution to clinical practitioners. It is imperative to inform the medical community of the clinical and radiological features, which may lead to misinterpretation of a glioblastoma as a vaccination-associated ADEM as well as warning against the over-diagnosis of vaccine-associated adverse events. This is of particular importance within the context of the current widespread vaccination efforts due to the COVID-19 pandemic and the related publication bias of findings linked to infection and vaccination.

Author contributions PW had full access to all of the data in the case report and takes responsibility for the integrity of the data and the accuracy of the data analysis. Concept and design: OS and W. Acquisition, analysis, or interpretation of data: all authors. Drafting of the manuscript: OS and W. Critical revision of the manuscript for important intellectual content: all authors.

Funding There was no funding to this study. 


\section{Declarations}

Conflicts of interest Ciara O'Sullivan has no conflict of interest. Fabian Zach has no conflict of interest. Tobias Moser has no conflict of interest. Georg Pilz has no conflict of interest. Andrea Harrer has no conflict of interest. Eugen Trinka has no conflict of interest. Christian Enzinger has no conflict of interest. Pfaff Johannes has no conflict of interest. Peter Wipfler has no conflict of interest.

Ethical approval All procedures performed in studies involving human participants were in accordance with the ethical standards of the institutional and/or national research committee and with the 1964 Helsinki declaration and its later amendments or comparable ethical standards.

\section{References}

1. COVID-19 weekly epidemiological update; Edition 44, (Published 15 June 2021)
2. Montalvan V, Lee J, Bueso T, De Toledo J, Rivas K (2020) Neurological manifestations of COVID-19 and other coronavirus infections: a systematic review. Clin Neurol Neurosurg 194:105921. https://doi.org/10.1016/j.clineuro.2020.105921

3. Novi G, Rossi T, Pedemonte E et al (2020) Acute disseminated encephalomyelitis after SARS-CoV-2 infection. Neurol Neuroimmunol Neuroinflamm 7:e797. https://doi.org/10.1212/NXI.00000 00000000797

4. Parsons T, Banks S, Bae C et al (2020) COVID-19-associated acute disseminated encephalomyelitis (ADEM). J Neurol 267:2799-2802. https://doi.org/10.1007/s00415-020-09951-9

5. Cao L, Ren L (2021) Acute disseminated encephalomyelitis after severe acute respiratory syndrome coronavirus 2 vaccination: a case report. Acta Neurol Belg. https://doi.org/10.1007/ s13760-021-01608-2

6. Hardy TA, Reddel SW, Barnett MH et al (2016) Atypical inflammatory demyelinating syndromes of the CNS. Lancet Neurol 15:967-981. https://doi.org/10.1016/S1474-4422(16)30043-6 